Research Article

\title{
Topological Properties of Nanostar Dendrimer and Smart Polymer
}

\author{
Muhammad Aamer Rashid (D), ${ }^{1}$ Sarfraz Ahmad, ${ }^{1}$ Murat Cancan $\mathbb{D D}^{2},{ }^{2}$ \\ and Mehwish Hussain Muhammad ${ }^{3}$ \\ ${ }^{1}$ Department of Mathematics, COMSATS University Islamabad, Lahore Campus, Lahore 54000, Pakistan \\ ${ }^{2}$ Faculty of Education, Van Yuzuncu Yil University, Van, Turkey \\ ${ }^{3}$ College of Chemistry and Molecular Engineering, Zhengzhou University, Zhengzhou, 450001, China
}

Correspondence should be addressed to Muhammad Aamer Rashid; aamerrasheed7869@gmail.com

Received 25 July 2019; Accepted 28 February 2020; Published 21 April 2020

Guest Editor: Shaohui Wang

Copyright (c) 2020 Muhammad Aamer Rashid et al. This is an open access article distributed under the Creative Commons Attribution License, which permits unrestricted use, distribution, and reproduction in any medium, provided the original work is properly cited.

\begin{abstract}
The nanostar dendrimers are a piece of another gathering of macromolecules that seem, by all accounts, to be photon pipes simply like counterfeit reception apparatuses. In addition, nanostar dendrimers are one of the fundamental stuffs of nanobiotechnology. The smart polymers are large-scale particles that show an emotional physioconcoction change because of little changes in their condition, for example, temperature, $\mathrm{pH}$, light, attractive field, and ionic variables. A topological record of a graph $G$ is a numeric quantity notorious with $G$ which portrays subatomic diagram $G$. In this paper, we decide first and second Zagreb indices, hyperZagreb index, first multiple Zagreb index, second numerous Zagreb index, and Zagreb polynomials for nanostar dendrimer and smart polymer.
\end{abstract}

\section{Introduction}

Scientific science is a part of hypothetical science where we examine and anticipate the compound erection by exploiting arithmetical devices. Substance diagram hypothesis is a part of scientific science wherein we apply apparatuses of chart hypothesis to display the synthetic marvel scientifically. This hypothesis contributes an unmistakable job in the fields of concoction sciences.

A nanostructure is an object of middle of the road size among minute and atomic structures. This is approximately due to a physical measurement lesser than 100 nanometers, extending from groups of iotas to dimensional layers. Nanobiotechnology is a hurriedly boosting territory of logical and mechanical open door that applies the apparatuses and procedures of nanofabrication to fabricate gadgets for examining biosystems.

From a polymer science perspective, dendrimers are almost immaculate monodisperse macromolecules with a customary and exceptionally fanned three-dimensional design. The nanostar dendrimer is a piece of another gathering of macroparticles that seem, by all accounts, to be photon channels simply like counterfeit reception apparatuses. These macromolecules and all those more absolutely containing phosphorus are utilized in the development of nanotubes, smaller scale macrocapsules, nanolatex, shaded glasses, concoction sensors, and adjusted terminals [1].

A topological index is a numeric amount related with a graph which portrays the topology of chart and is invariant under diagram automorphism. A topological record Top $(G)$ of a graph $G$ is a number with the property that, for each chart $H$ isomorphic to $G$, Top $(H)=\operatorname{Top}(G)$. The idea of topological record originated from work done by Wiener [2], while he was taking a shot at breaking point of paraffin. He named this record as way number. Later on, the way number was renamed as Wiener index. The Wiener list is the first and most concentrated topological file, both from hypothetical perspective and applications, and characterized as the whole of separations between all sets of vertices in $G$, see $[3,4]$ for subtleties. 
One of the most seasoned topological files is the primary Zagreb list presented by Gutman and Trinajstic in 1972 [5] based on the level of vertices of $G$, and the first and second Zagreb indices are characterized as

$$
\begin{aligned}
& M_{1}(G)=\sum_{\widehat{e} f \in E(G)}[\Omega(\widehat{e})+\widehat{f}], \\
& M_{2}(G)=\sum_{\widehat{e} f \in E(G)}[\Omega(\widehat{e}) \times \Omega(\widehat{f})] .
\end{aligned}
$$

In 2013, Shirdel et al. [6] introduced a new degree-based Zagreb index named "hyper-Zagreb index" as

$$
H M(G)=\sum_{\widehat{e} f \in E(G)}[\Omega(\widehat{e})+\Omega(\widehat{f})]^{2} .
$$

In 2012, Ghorbani and Azimi [7] characterized two new forms of Zagreb lists of a diagram $G$. The first multiple Zagreb index $P M_{1}(G)$, the second multiple Zagreb index $P M_{2}(G)$, and these are characterized as

$$
\begin{aligned}
& P M_{1}(G)=\prod_{\widehat{e} f \in E(G)}[\Omega(\widehat{e})+\Omega(\widehat{f})], \\
& P M_{2}(G)=\prod_{\widehat{e f f} \in E(G)}[\Omega(\widehat{e})+\Omega(\widehat{f})] .
\end{aligned}
$$

In [8], the Zagreb polynomials are characterized as

$$
\begin{aligned}
& M_{1}(G, x)=\sum_{\widehat{e} f \in E(G)} x^{[\Omega(\widehat{e})+\Omega(\widehat{f})]}, \\
& M_{2}(G, x)=\sum_{\widehat{e} f \in E(G)} x^{[\Omega(\widehat{e})+\Omega(\widehat{f})]} .
\end{aligned}
$$

For further investigation of topological files of different chart families, see $[5,6,9-23]$.

\section{Nanostar Dendrimers $N_{S} C_{5} C_{6}[n]$ and $N S D[n]$}

Nanostar dendrimers are one of the fundamental objects of nanobiotechnology. They have an all-around characterized subatomic topology. Their progression insightful development pursues a scientific movement. In a precise expression, nanostar dendrimers are hyperbranched macromolecules, demonstrating a thorough, tastefully engaging engineering. The nanostar dendrimer is a piece of another gathering of macromolecules having extraordinary applications [1]. In 2012, Rostamia et al. [24] figured first geometric-number juggling list, Randic file, and entirely available file for nanostar dendrimer $N_{5} C_{5} C_{6}[n]$ and $N S D[n]$. In this segment, we process first and Second Zagreb lists, hyper-Zagreb list, first different Zagreb record, second various Zagreb file, and Zagreb polynomials for nanostar dendrimers $\mathrm{NSC}_{5} \mathrm{C}_{6}[n]$ and $\mathrm{NSD}[n]$.

\subsection{Methodology and Construction of Nanostar Dendrimers} $\mathrm{NSC}_{5} \mathrm{C}_{6}[n]$ Formulas. Consider the atomic chart $N_{S C_{5}} C_{6}[n]$, where $n$ are ventures of development in this kind of nanostar dendrimer (see Figure 1). It is anything but difficult to compute that the quantity of vertices in $N \mathrm{NC}_{5} \mathrm{C}_{6}[n]$ is $9 \cdot 2^{n+2}-44$ and the quantity of edges is $10 \cdot 2^{n+2}-50$, see likewise [24].

Moreover, there are seven types of edges in $N_{S} C_{5} C_{6}[n]$. To compute the above results, we define seven partitions of edge set $E\left(N_{S C} C_{6}[n]\right)$ and compute their cardinalities in the following way:

$$
\begin{aligned}
& E_{1}\left(N S C_{5} C_{6}[n]\right)=\left\{\widehat{e} \hat{f} \in E\left(N S C_{5} C_{6}[n]\right) \mid \Omega(\widehat{e})=1, \Omega(\widehat{f})=3\right\}, \\
&\left|E_{1}\left(N S C_{5} C_{6}[n]\right)\right|=2^{n+2}-6, \\
& E_{2}\left(N S C_{5} C_{6}[n]\right)=\left\{\widehat{e} \hat{f} \in E\left(N S C_{5} C_{6}[n]\right) \mid \Omega(\widehat{e})=1, \Omega(\widehat{f})=4\right\}, \\
&\left|E_{2}\left(N S C_{5} C_{6}[n]\right)\right|=2^{n+2}, \\
& E_{3}\left(N S C_{5} C_{6}[n]\right)=\left\{\widehat{e} \hat{f} \in E\left(N S C_{5} C_{6}[n]\right) \mid \Omega(\widehat{e})=2, \Omega(\widehat{f})=2\right\}, \\
&\left|E_{3}\left(N S C_{5} C_{6}[n]\right)\right|=2^{n+2}-6, \\
& E_{4}\left(N S C_{5} C_{6}[n]\right)=\left\{\widehat{e} \widehat{f} \in E\left(N S C_{5} C_{6}[n]\right) \mid \Omega(\widehat{e})=2, \Omega(\widehat{f})=3\right\}, \\
&\left|E_{4}\left(N S C_{5} C_{6}[n]\right)\right|=9 \cdot 2^{n+2}-28, \\
& E_{5}\left(N S C_{5} C_{6}[n]\right)=\left\{\widehat{e} \hat{f} \in E\left(N S C_{5} C_{6}[n]\right) \mid \Omega(\widehat{e})=2, \Omega(\widehat{f})=4\right\}, \\
&\left|E_{5}\left(N S C_{5} C_{6}[n]\right)\right|=2^{n+1}, \\
& E_{6}\left(N S C_{5} C_{6}[n]\right)=\left\{\widehat{e} \widehat{f} \in E\left(N S C_{5} C_{6}[n]\right) \mid \Omega(\widehat{e})=3, \Omega(\widehat{f})=3\right\}, \\
&\left|E_{6}\left(N S C_{5} C_{6}[n]\right)\right|=3 \cdot 2^{n}-10, \\
& E_{7}\left(N S C_{5} C_{6}[n]\right)=\left\{\widehat{e} \widehat{f} \in E\left(N S C_{5} C_{6}[n]\right) \mid \Omega(\widehat{e})=4, \Omega(\widehat{f})=4\right\}, \\
&\left|E_{7}\left(N S C_{5} C_{6}[n]\right)\right|=2^{n} .
\end{aligned}
$$




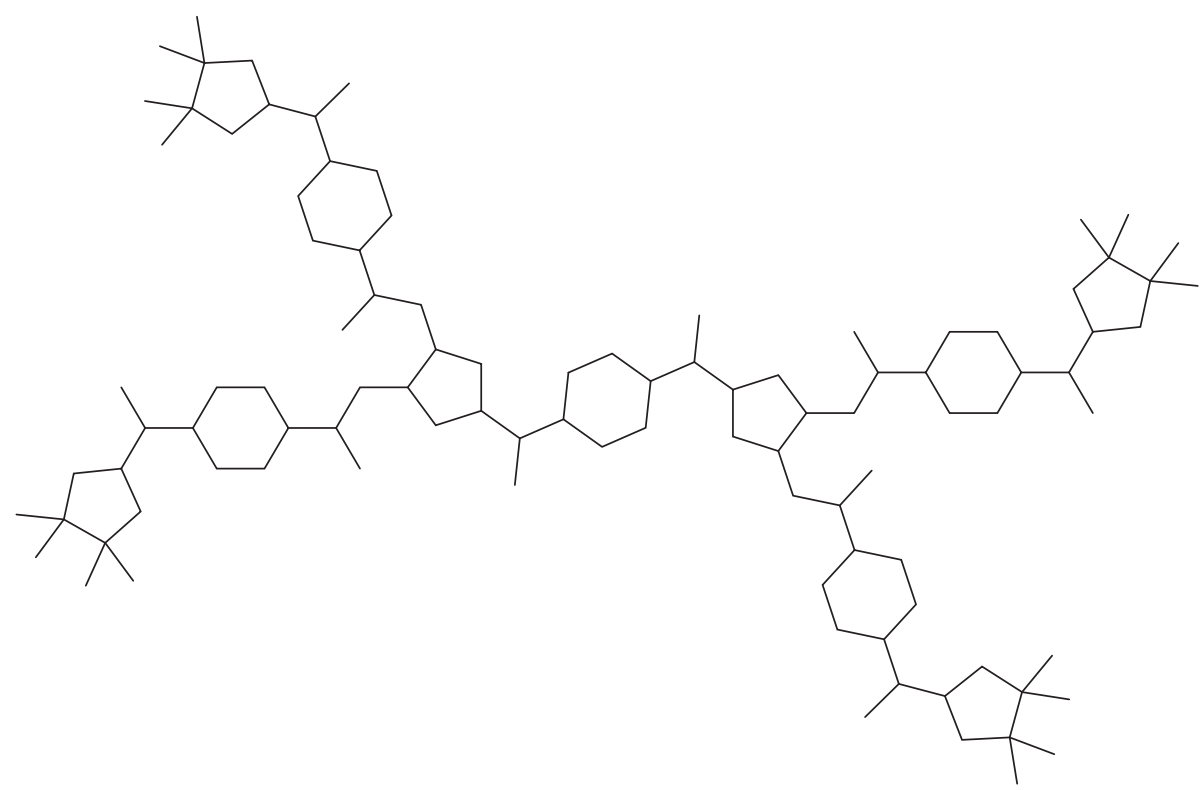

Figure 1: The nanostar dendrimer $\mathrm{NSC}_{5} \mathrm{C}_{6}$ [2].

Now using equations (1)-(7), we have the following 2.1.1. First and Second Zagreb Index results.

$$
\begin{aligned}
& M_{1}(G)=\sum_{\widehat{e} f \in E(G)}[\Omega(\widehat{e})+\Omega(\widehat{f})], \\
& M_{1}\left(N S C_{5} C_{6}[n]\right)=\sum_{\widehat{e} \hat{f} \in E_{1}}[\Omega(\widehat{e})+\Omega(\widehat{f})]+\sum_{\widehat{e} f \in E_{2}}[\Omega(\widehat{e})+\Omega(\widehat{f})]+\sum_{\widehat{e} \hat{f} \in E_{3}}[\Omega(\widehat{e})+\Omega(\widehat{f})] \\
& +\sum_{\widehat{e} f \in E_{4}}[\Omega(\widehat{e})+\Omega(\hat{f})]+\sum_{\widehat{e f} \in E_{5}}[\Omega(\widehat{e})+\Omega(\hat{f})]+\sum_{\widehat{e} f \in E_{6}}[\Omega(\widehat{e})+\Omega(\widehat{f})]+\sum_{\widehat{e} f \in E_{7}}[\Omega(\widehat{e})+\Omega(\hat{f})] \\
& =4\left|E_{1}\right|+5\left|E_{2}\right|+4\left|E_{3}\right|+5\left|E_{4}\right|+6\left|E_{5}\right|+6\left|E_{6}\right|+8\left|E_{7}\right| \\
& =4\left(2^{n+2}-6\right)+5\left(2^{n+2}\right)+4\left(2^{n+2}-6\right)+5\left(9 \cdot 2^{n+2}-28\right)+6\left(2^{n+1}\right)+6\left(3 \cdot 2^{n}-10\right)+8\left(2^{n}\right) \text {, } \\
& M_{2}(G)=\sum_{\widehat{e} f \in E(G)}[\Omega(\widehat{e}) \times \Omega(\widehat{f})], \\
& M_{2}\left(N S C_{5} C_{6}[n]\right)=\sum_{\widehat{e} \hat{f} \in E_{1}}[\Omega(\widehat{e}) \times \Omega(\hat{f})]+\sum_{\widehat{e} \hat{f} \in E_{2}}[\Omega(\widehat{e}) \times \Omega(\hat{f})]+\sum_{\widehat{e} \hat{f} \in E_{3}}[\Omega(\widehat{e}) \times \Omega(\hat{f})] \\
& +\sum_{\widehat{e} f \in E_{4}}[\Omega(\widehat{e}) \times \Omega(\hat{f})]+\sum_{\widehat{e} \hat{f} \in E_{5}}[\Omega(\widehat{e}) \times \Omega(\hat{f})]+\sum_{\widehat{e} f \in E_{6}}[\Omega(\widehat{e}) \times \Omega(\widehat{f})]+\sum_{\widehat{e} f \in E_{7}}[\Omega(\widehat{e}) \times \Omega(\hat{f})] \\
& =3\left|E_{1}\right|+4\left|E_{2}\right|+4\left|E_{3}\right|+6\left|E_{4}\right|+8\left|E_{5}\right|+9\left|E_{6}\right|+16\left|E_{7}\right| \\
& =3\left(2^{n+2}-6\right)+4\left(2^{n+2}\right)+4\left(2^{n+2}-6\right)+6\left(9 \cdot 2^{n+2}-28\right)+8\left(2^{n+1}\right)+9\left(3 \cdot 2^{n}-10\right)+16\left(2^{n}\right) \text {. }
\end{aligned}
$$


2.1.2. Hyper-Zagreb Index

$$
\begin{aligned}
H M(G)= & \sum_{\widehat{e} \hat{f} \in E(G)}[\Omega(\widehat{e})+\Omega(\widehat{f})]^{2}, \\
H M\left(N S C_{5} C_{6}[n]\right)= & \sum_{\widehat{e} \widehat{f} \in E_{1}}[\Omega(\widehat{e})+\Omega(\widehat{f})]^{2}+\sum_{\widehat{e} \hat{e} \in E_{2}}[\Omega(\widehat{e})+\Omega(\widehat{f})]^{2}+\sum_{\widehat{e} \widehat{f} \in E_{3}}[\Omega(\widehat{e})+\Omega(\widehat{f})]^{2} \\
& +\sum_{\widehat{e} \hat{f} \in E_{4}}[\Omega(\widehat{e})+\Omega(\widehat{f})]^{2}+\sum_{\widehat{e} \hat{f} \in E_{5}}[\Omega(\widehat{e})+\Omega(\widehat{f})]^{2}+\sum_{\widehat{e} \widehat{f} \in E_{6}}[\Omega(\widehat{e})+\Omega(\widehat{f})]^{2}+\sum_{\widehat{e} \widehat{f} \in E_{7}}[\Omega(\widehat{e})+\Omega(\widehat{f})]^{2} \\
= & 16\left|E_{1}\right|+25\left|E_{2}\right|+16\left|E_{3}\right|+25\left|E_{4}\right|+36\left|E_{5}\right|+36\left|E_{6}\right|+64\left|E_{7}\right| \\
= & 16\left(2^{n+2}-6\right)+25\left(2^{n+2}\right)+16\left(2^{n+2}-6\right)+25\left(9 \cdot 2^{n+2}-28\right)+36\left(2^{n+1}\right)+36\left(3 \cdot 2^{n}-10\right)+64\left(2^{n}\right) .
\end{aligned}
$$

\subsubsection{First and Second Multiple Zagreb Index}

$$
\begin{aligned}
& P M_{1}(G)=\prod_{\widehat{e} \hat{f} \in E(G)}[\Omega(\widehat{e})+\Omega(\widehat{f})], \\
& P M_{1}\left(N S C_{5} C_{6}[n]\right)=\prod_{\widehat{e} \widehat{f} \in E_{1}}[\Omega(\widehat{e})+\Omega(\widehat{f})] \times \prod_{\widehat{e} \hat{f} \in E_{2}}[\Omega(\widehat{e})+\Omega(\widehat{f})] \times \prod_{\widehat{e} \widehat{e} \in E_{3}}[\Omega(\widehat{e})+\Omega(\widehat{f})] \\
& \times \prod_{\widehat{e} \widehat{f} \in E_{4}}[\Omega(\widehat{e})+\Omega(\widehat{f})] \times \prod_{\widehat{e} \widehat{f} \in E_{5}}[\Omega(\widehat{e})+\Omega(\widehat{f})] \times \prod_{\widehat{e} \hat{e} \in E_{6}}[\Omega(\widehat{e})+\Omega(\widehat{f})] \times \prod_{\widehat{e} \widehat{f} \in E_{7}}[\Omega(\widehat{e})+\Omega(\widehat{f})] \\
& =4^{\left|E_{1}\right|} \times 5^{\left|E_{2}\right|} \times 4^{\left|E_{3}\right|} \times 5^{\left|E_{4}\right|} \times 6^{\left|E_{5}\right|} \times 6^{\left|E_{6}\right|} \times 8^{\left|E_{7}\right|} \\
& =4^{\left(2^{n+2}-6\right)} \times 5^{\left(2^{n+2}\right)} \times 4^{\left(2^{n+2}-6\right)} \times 5^{\left(9 \cdot 2^{n+2}-28\right)} \times 6^{\left(2^{n+1}\right)} \times 6^{\left(3 \cdot 2^{n}-10\right)} \times 8^{\left(2^{n}\right)}, \\
& P M_{2}(G)=\prod_{\widehat{e} \hat{f} \in E(G)}[\Omega(\widehat{e}) \times \Omega(\hat{f})] \\
& P M_{2}\left(N S C_{5} C_{6}[n]\right)=\prod_{\widehat{e} \hat{f} \in E_{1}}[\Omega(\widehat{e}) \times \Omega(\widehat{f})] \times \prod_{\widehat{e} \hat{f} \in E_{2}}[\Omega(\widehat{e}) \times \Omega(\widehat{f})] \times \prod_{\widehat{e} \hat{e} \in E_{3}}[\Omega(\widehat{e}) \times \Omega(\widehat{f})] \\
& \times \prod_{\widehat{e} \widehat{f} \in E_{4}}[\Omega(\widehat{e}) \times \Omega(\widehat{f})] \times \prod_{\widehat{e} \widehat{f} \in E_{5}}[\Omega(\widehat{e}) \times \Omega(\widehat{f})] \times \prod_{\widehat{e} \hat{e} \in E_{6}}[\Omega(\widehat{e}) \times \Omega(\widehat{f})] \times \prod_{\widehat{e} \widehat{f} \in E_{7}}[\Omega(\widehat{e}) \times \Omega(\widehat{f})] \\
& =3^{\left|E_{1}\right|} \times 4^{\left|E_{2}\right|} \times 4^{\left|E_{3}\right|} \times 6^{\left|E_{4}\right|} \times 8^{\left|E_{5}\right|} \times 9^{\left|E_{6}\right|} \times 16^{\left|E_{7}\right|} \\
& =3^{\left(2^{n+2}-6\right)} \times 4^{\left(2^{n+2}\right)} \times 4^{\left(2^{n+2}-6\right)} \times 6^{\left(9 \cdot 2^{n+2}-28\right)} \times 8^{\left(2^{n+1}\right)} \times 9^{\left(3 \cdot 2^{n}-10\right)} \times 16^{\left(2^{n}\right)} .
\end{aligned}
$$


2.1.4. First and Second Zagreb Polynomial

$$
\begin{aligned}
& M_{1}(G, x)=\sum_{\widehat{e} \widehat{f} \in E(G)} x^{[\Omega(\widehat{e})+\Omega(\widehat{f})]}, \\
& M_{1}\left(N S C_{5} C_{6}[n], x\right)=\sum_{\widehat{e} \widehat{f} \in E_{1}} x^{[\Omega(\widehat{e})+\Omega(\widehat{f})]}+\sum_{\widehat{e} \widehat{f} \in E_{2}} x^{[\Omega(\widehat{e})+\Omega(\widehat{f})]}+\sum_{\widehat{e} \widehat{f} \in E_{3}} x^{[\Omega(\widehat{e})+\Omega(\widehat{f})]} \\
& +\sum_{\widehat{e} \widehat{f} \in E_{4}} x^{[\Omega(\widehat{e})+\Omega(\widehat{f})]}+\sum_{\widehat{e} \widehat{f} \in E_{5}} x^{[\Omega(\widehat{e})+\Omega(\widehat{f})]}+\sum_{\widehat{e} \widehat{f} \in E_{6}} x^{[\Omega(\widehat{e})+\Omega(\widehat{f})]}+\sum_{\widehat{e} \widehat{f} \in E_{7}} x^{[\Omega(\widehat{e})+\Omega(\widehat{f})]} \\
& =\left|E_{1}\right| x^{4}+\left|E_{2}\right| x^{5}+\left|E_{3}\right| x^{4}+\left|E_{4}\right| x^{5}+\left|E_{5}\right| x^{6}+\left|E_{6}\right| x^{6}+\left|E_{7}\right| x^{8} \\
& =\left(2^{n+2}-6\right) x^{4}+\left(2^{n+2}\right) x^{5}+\left(2^{n+2}-6\right) x^{4}+\left(9 \cdot 2^{n+2}-28\right) x^{5}+\left(2^{n+1}\right) x^{6}+\left(3 \cdot 2^{n}-10\right) x^{6}+\left(2^{n}\right) x^{8} \text {, } \\
& M_{2}(G, x)=\sum_{\widehat{e} \widehat{f} \in E(G)} x^{[\Omega(\widehat{e}) \times \Omega(\widehat{f})]}, \\
& M_{2}\left(N S C_{5} C_{6}[n], x\right)=\sum_{\widehat{e} \widehat{f} \in E_{1}} x^{[\Omega(\widehat{e}) \times \Omega(\widehat{f})]}+\sum_{\widehat{e} \widehat{f} \in E_{2}} x^{[\Omega(\widehat{e}) \times \Omega(\widehat{f})]}+\sum_{\widehat{e} \widehat{f} \in E_{3}} x^{[\Omega(\widehat{e}) \times \Omega(\widehat{f})]} \\
& +\sum_{\widehat{e} \widehat{f} \in E_{4}} x^{[\Omega(\widehat{e}) \times \Omega(\widehat{f})]}+\sum_{\widehat{e} \widehat{f} \in E_{5}} x^{[\Omega(\widehat{e}) \times \Omega(\widehat{f})]}+\sum_{\widehat{e} \widehat{f} \in E_{6}} x^{[\Omega(\widehat{e}) \times \Omega(\widehat{f})]}+\sum_{\widehat{e} \widehat{f} \in E_{7}} x^{[\Omega(\widehat{e}) \times \Omega(\widehat{f})]} \\
& =\left|E_{1}\right| x^{3}+\left|E_{2}\right| x^{4}+\left|E_{3}\right| x^{4}+\left|E_{4}\right| x^{6}+\left|E_{5}\right| x^{8}+\left|E_{6}\right| x^{9}+\left|E_{7}\right| x^{16} \\
& =\left(2^{n+2}-6\right) x^{3}+\left(2^{n+2}\right) x^{4}+\left(2^{n+2}-6\right) x^{4}+\left(9 \cdot 2^{n+2}-28\right) x^{6}+\left(2^{n+1}\right)^{8}+\left(3 \cdot 2^{n}-10\right) x^{9}+\left(2^{n}\right) x^{16} \text {. }
\end{aligned}
$$

2.2. Graphical Representation and Discussion of Results. In Figure 2(a), comparison of $M_{1}\left(N_{S} C_{5} C_{6}[n], x\right)$ and $M_{2}\left(N_{S} C_{5} C_{6}[n], x\right)$ polynomials of $N C_{5} C_{6}[n]$ is given with $M_{1}\left(N_{S} C_{5} C_{6}[n], x\right)$ in red and $M_{2}\left(N_{S C} C_{5} C_{6}[n], x\right)$ in blue. In Figure 2(b), we compare the indices $P M_{1}\left(N S C_{5} C_{6}[n]\right)$ and $P_{2}\left(N_{5} C_{5} C_{6}[n]\right)$ with $P M_{1}\left(N_{S} C_{5} C_{6}[n]\right)$ in red and $\mathrm{PM}_{2}\left(\mathrm{NSC}_{5} \mathrm{C}_{6}[n]\right)$ in blue. In Figure 2(c), we compare the indices $\quad M_{1}\left(N_{S C} C_{5} C_{6}[n]\right), \quad M_{2}\left(N_{S C} C_{5} C_{6}[n]\right), \quad$ and $H M\left(N_{5} C_{5} C_{6}[n]\right)$ with $M_{1}\left(N_{S S} C_{5} C_{6}[n]\right)$ in red, $\mathrm{PM}_{2}\left(\mathrm{NSC}_{5} \mathrm{C}_{6}[n]\right)$ in blue, and $\mathrm{HM}\left(\mathrm{NSC}_{5} \mathrm{C}_{6}[n]\right)$ in black. It merits referencing that above-plotted charts demonstrate the reliance of each topological list on $n$. From these figures, one can envision that each topological file acts uniquely in contrast to other against parameters.

\subsection{Methodology and Construction of Nanostar Dendrimers} $N S D[n]$ Formulas. Consider the subatomic diagram $N S D[n]$, where $n$ are ventures of development in this sort of nanostar dendrimer (see Figure 3). It is anything but difficult to ascertain that the quantity of vertices in $N S D[n]$ is 120 . $2^{n}-108$ and the quantity of edges is $140 \cdot 2^{n}-127$, see likewise [24]. Moreover, there are three types of edges in $N S D[n]$ based on degrees of end vertices of each edge. To compute the above results, we define three partitions of edge set $E(N S D[n])$ and compute their cardinalities in the following way:

$$
\begin{aligned}
E_{1}(N S D[n]) & =\{\widehat{e} \hat{f} \in E(N S D[n]) \mid \Omega(\widehat{e})=2, \Omega(\widehat{f})=2\}, \\
\left|E_{1}(N S D[n])\right| & =52 \cdot 2^{n}-48, \\
E_{2}(N S D[n]) & =\{\widehat{e} \widehat{f} \in E(N S D[n]) \mid \Omega(\widehat{e})=2, \Omega(\widehat{f})=3\}, \\
\left|E_{2}(N S D[n])\right| & =48 \cdot 2^{n}-44, \\
E_{3}(N S D[n]) & =\{\hat{e} \hat{f} \in E(N S D[n]) \mid \Omega(\widehat{e})=3, \Omega(\widehat{f})=3\}, \\
\left|E_{3}(N S D[n])\right| & =36 \cdot 2^{n}-35 .
\end{aligned}
$$

Now using equations (1)-(7), we have the following results. 


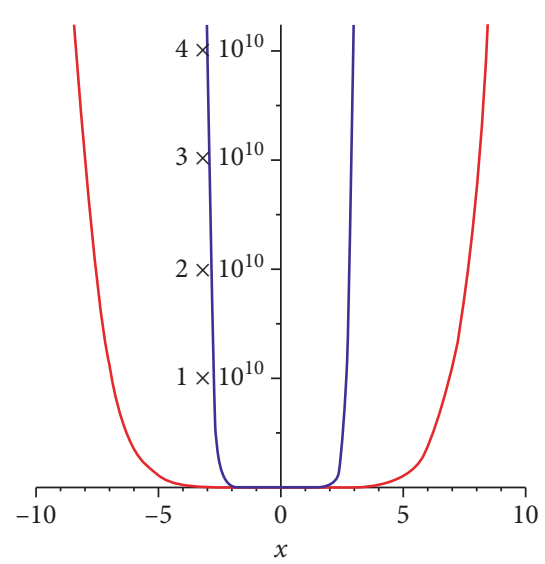

(a)

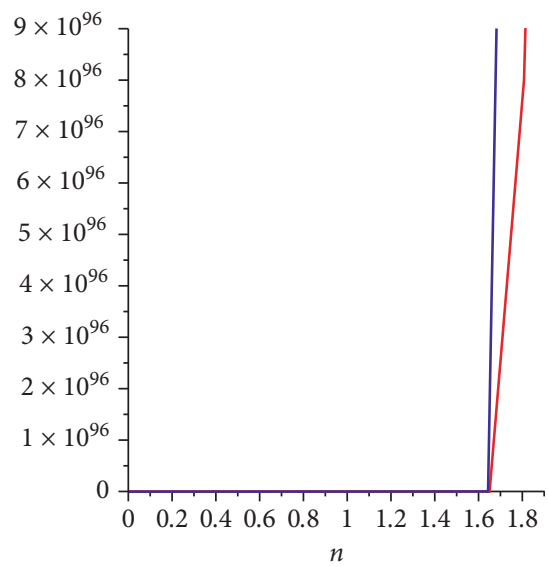

(b)

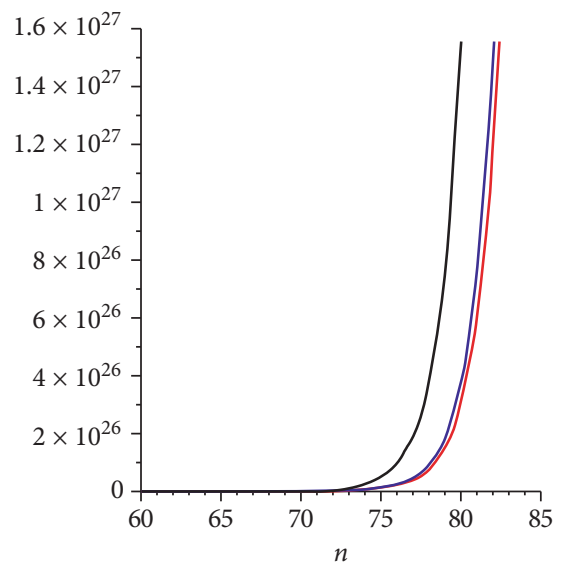

(c)

Figure 2: Comparison of topological indices and polynomials for $N_{S} C_{5} C_{6}[n]$.

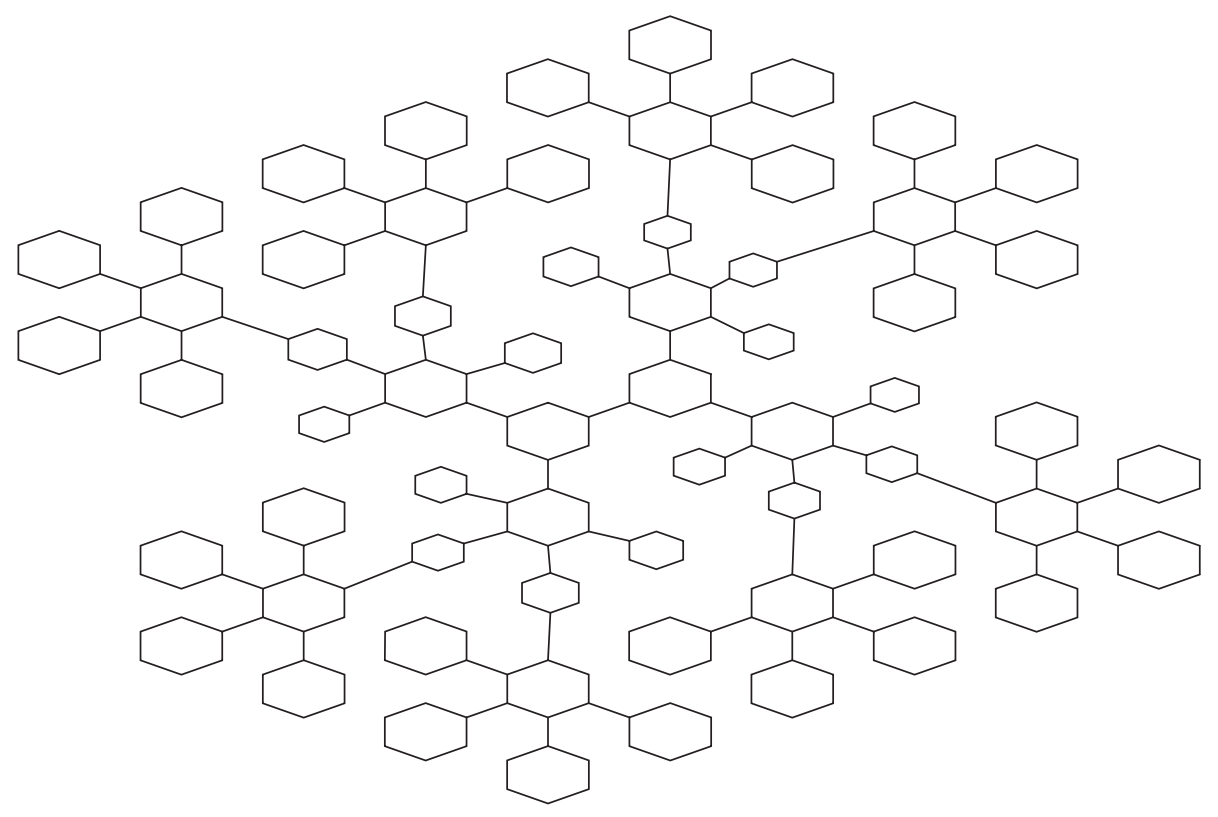

FIgURE 3: The nanostar dendrimer NS $D[n]$.

2.3.1. First and Second Zagreb Index

$$
\begin{aligned}
M_{1}(G) & =\sum_{\widehat{e} f \in E(G)}[\Omega(\widehat{e})+\Omega(\widehat{f})], \\
M_{1}(N S D[n]) & =\sum_{\widehat{e} \widehat{f} \in E_{1}}[\Omega(\widehat{e})+\Omega(\widehat{f})]+\sum_{\widehat{e} \widehat{f} \in E_{2}}[\Omega(\widehat{e})+\Omega(\widehat{f})]+\sum_{\widehat{e} \widehat{f} \in E_{3}}[\Omega(\widehat{e})+\Omega(\widehat{f})] \\
& =4\left|E_{1}\right|+5\left|E_{2}\right|+6\left|E_{3}\right|=4\left(52 \cdot 2^{n}-48\right)+5\left(48 \cdot 2^{n}-44\right)+6\left(36 \cdot 2^{n}-35\right), \\
M_{2}(G) & =\sum_{\widehat{e} \widehat{f} \in E(G)}[\Omega(\widehat{e})+\Omega(\widehat{f})], \\
M_{2}(N S D[n]) & =\sum_{\widehat{e} \widehat{f} \in E_{1}}[\Omega(\widehat{e})+\Omega(\widehat{f})]+\sum_{\widehat{e} \widehat{f} \in E_{2}}[\Omega(\widehat{e})+\Omega(\widehat{f})]+\sum_{\widehat{e} \widehat{f} \in E_{3}}[\Omega(\widehat{e})+\Omega(\widehat{f})] \\
& =4\left|E_{1}\right|+6\left|E_{2}\right|+9\left|E_{3}\right|=4\left(52 \cdot 2^{n}-48\right)+6\left(48 \cdot 2^{n}-44\right)+9\left(36 \cdot 2^{n}-35\right) .
\end{aligned}
$$


2.3.2. Hyper-Zagreb Index

$$
\begin{aligned}
H M(G) & =\sum_{\widehat{e} \widehat{f} \in E(G)}[\Omega(\widehat{e})+\Omega(\widehat{f})]^{2}, \\
H M(N S D[n]) & =\sum_{\widehat{e} \widehat{f} \in E_{1}}[\Omega(\widehat{e})+\Omega(\widehat{f})]^{2}+\sum_{\widehat{e} \widehat{f} \in E_{2}}[\Omega(\widehat{e})+\Omega(\widehat{f})]^{2}+\sum_{\widehat{e} \widehat{f} \in E_{3}}[\Omega(\widehat{e})+\Omega(\widehat{f})]^{2} \\
& =16\left|E_{1}\right|+25\left|E_{2}\right|+36\left|E_{3}\right|=16\left(52 \cdot 2^{n}-48\right)+25\left(48 \cdot 2^{n}-44\right)+36\left(36 \cdot 2^{n}-35\right) .
\end{aligned}
$$

\subsubsection{First and Second Multiple Zagreb Index}

$$
\begin{aligned}
P M_{1}(G) & =\prod_{\widehat{e} \hat{f} \in E(G)}[\Omega(\widehat{e})+\Omega(\widehat{f})], \\
P M_{1}(N S D[n]) & =\prod_{\widehat{e} \hat{f} \in E_{1}}[\Omega(\widehat{e})+\Omega(\widehat{f})]+\prod_{\widehat{e} \widehat{f} \in E_{2}}[\Omega(\widehat{e})+\Omega(\widehat{f})]+\prod_{\widehat{e} \hat{f} \in E_{3}}[\Omega(\widehat{e})+\Omega(\widehat{f})] \\
& =4^{\left|E_{1}\right|} \times 5^{\left|E_{2}\right|} \times 6^{\left|E_{3}\right|}=4^{\left(52 \cdot 2^{n}-48\right)} \times 5^{\left(48 \cdot 2^{n}-44\right)} \times 6^{\left(36 \cdot 2^{n}-35\right)}, \\
P M_{2}(G) & =\prod_{\widehat{e} \widehat{f} \in E(G)}[\Omega(\widehat{e})+\Omega(\widehat{f})], \\
P M_{2}(N S D[n]) & =\prod_{\widehat{e} \hat{f} \in E_{1}}[\Omega(\widehat{e})+\Omega(\widehat{f})]+\prod_{\widehat{e} \widehat{f} \in E_{2}}[\Omega(\widehat{e})+\Omega(\widehat{f})]+\prod_{\widehat{e} \hat{f} \in E_{3}}[\Omega(\widehat{e})+\Omega(\widehat{f})] \\
& =4^{\left|E_{1}\right|} \times 6^{\left|E_{2}\right|} \times 9^{\left|E_{3}\right|}=4^{\left(52 \cdot 2^{n}-48\right)} \times 6^{\left(48 \cdot 2^{n}-44\right)} \times 9^{\left(36 \cdot 2^{n}-35\right)} .
\end{aligned}
$$

2.3.4. First and Second Zagreb Polynomial

$$
\begin{aligned}
M_{1}(G, x) & =\sum_{\widehat{e} f \in E(G)} x^{[\Omega(\widehat{e})+\Omega(\widehat{f})]}, \\
M_{1}(N S D[n], x) & =\sum_{\widehat{e} \hat{f} \in E_{1}} x^{[\Omega(\widehat{e})+\Omega(\widehat{f})]}+\sum_{\widehat{e} \widehat{f} \in E_{2}} x^{[\Omega(\widehat{e})+\Omega(\widehat{f})]}+\sum_{\widehat{e} \widehat{f} \in E_{3}} x^{[\Omega(\widehat{e})+\Omega(\widehat{f})]} \\
& =\left|E_{1}\right| x^{4}+\left|E_{2}\right| x^{5}+\left|E_{3}\right| x^{6}=\left(52 \cdot 2^{n}-48\right) x^{4}+\left(48 \cdot 2^{n}-44\right) x^{5}+\left(36 \cdot 2^{n}-35\right) x^{6}, \\
M_{2}(G, x) & =\sum_{\widehat{e} \widehat{f} \in E(G)} x^{[\Omega(\widehat{e})+\Omega(\widehat{f})]}, \\
M_{2}(N S D[n], x) & =\sum_{\widehat{e} \widehat{f} \in E_{1}} x^{[\Omega(\widehat{e})+\Omega(\widehat{f})]}+\sum_{\widehat{e} \widehat{f} \in E_{2}} x^{[\Omega(\widehat{e})+\Omega(\widehat{f})]}+\sum_{\widehat{e} \widehat{f} \in E_{3}} x^{[\Omega(\widehat{e})+\Omega(\widehat{f})]} \\
& =\left|E_{1}\right| x^{4}+\left|E_{2}\right| x^{6}+\left|E_{3}\right| x^{9}=\left(52 \cdot 2^{n}-48\right) x^{4}+\left(48 \cdot 2^{n}-44\right) x^{6}+\left(36 \cdot 2^{n}-35\right) x^{9} .
\end{aligned}
$$

2.4. Graphical Representation and Discussion of Results. In Figure 4(a), comparison of $M_{1}(N S D[n], x)$ and $M_{2}(N S D$ $[n], x)$ polynomials of $N S D[n]$ is given with $M_{1}(N S D[n], x)$ in red and $M_{2}(N S D[n], x)$ in blue. In Figure 4(b), we compare the indices $P M_{1}(N S D[n])$ and $P M_{2}(N S D[n])$ with $P M_{1}(N S D[n])$ in red and $P M_{2}(N S D[n])$ in blue. In
Figure 4(c), we compare the indices $M_{1}(N S D[n]), M_{2}$ (NSD $[n])$, and $H M(N S D[n])$ with $M_{1}(N S D[n])$ in red, $P M_{2}$ $(N S D[n])$ in blue, and $H M(N S D[n])$ in black. These figures also give us some extreme values of the certain topological index. Moreover, these graphs give an insight view to control the values of topological indices with $m$ and $n$. 


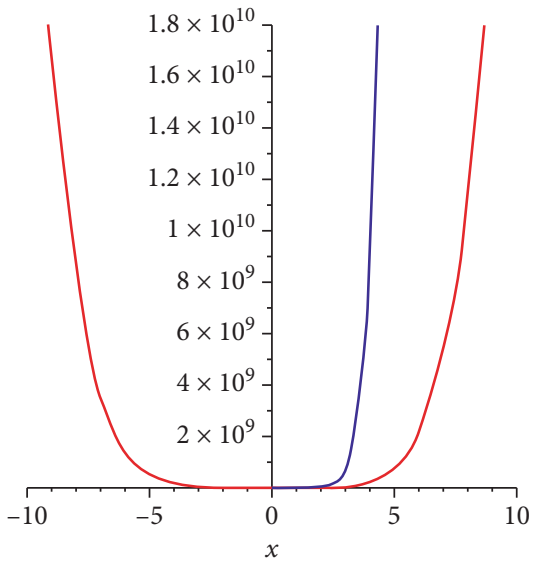

(a)

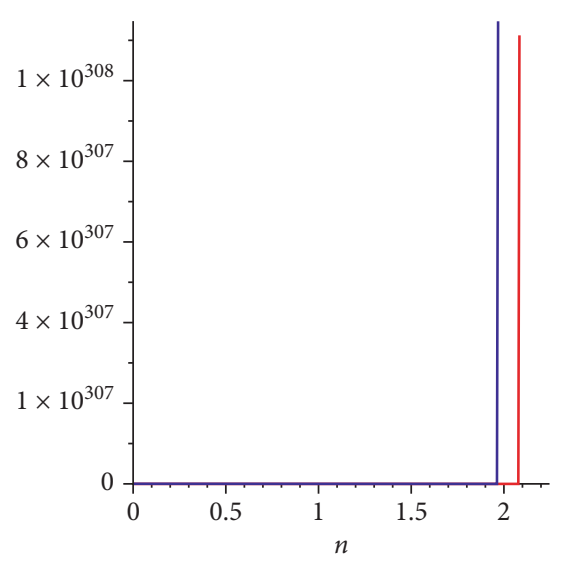

(b)

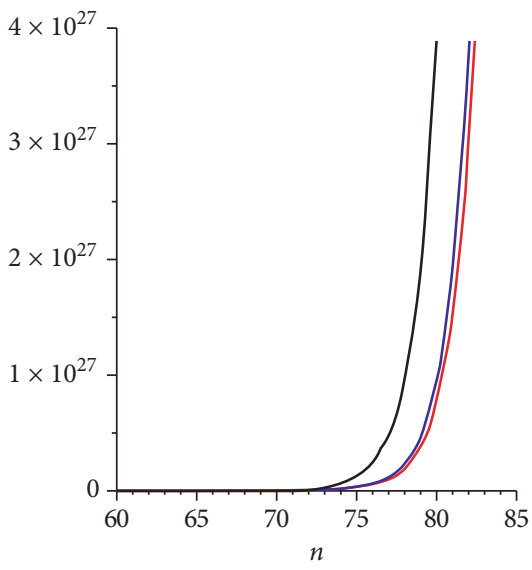

(c)

FIgURE 4: Comparison of topological indices and polynomials for $N S D[n]$.

\section{Smart Polymer $S P[n]$}

Keen polymers are characterized as the macromolecules that show a sensational physiochemical change because of little changes in their condition, for example, temperature, $\mathrm{pH}$, light, attractive field, and ionic variables [25]. Shrewd polymers are additionally called as upgrades responsive or smart or naturally responsive frameworks. Shrewd polymers have different applications in biomedical field as conveyance frameworks like brilliant polymers with protein or nucleic corrosive conveyance to intracellular targets, for example, ribosome or core and in tissue designing [26,27]. Polymeric micelles are one of the sorts of savvy polymers, which is accustomed to conveying hostile to malignant growth tranquilize, for instance, Dox-conjugated PEG-b-poly (aspartate) (PEG-PAsp) square copolymers [28]. In 2012, Shettya et al. [29] figured Randic record, first and second Zagreb lists, Geometric-math list, and atomic bond connectivity file of brilliant polymer.

In this area, we register hyper-Zagreb list, first various Zagreb file, second numerous Zagreb list, and Zagreb polynomials for the class of the shrewd polymer Doxstacked micelle including PEG-PAsp square copolymer with artificially conjugated Dox $S P[n]$ (see Figure 5).

3.1. Methodology and Construction of Smart Polymer $S P[n]$ Formulas. Consider the atomic chart $S P[n]$, where $n$ are ventures of development in this sort of polymers (see Figure 5). It is anything but difficult to ascertain that the quantity of vertices in $S P[n]$ is $49 n+6$ and the quantity of edges is $54 n+5$, see likewise [29]. Moreover, there are eight types of edges in $S P[n]$ based on degrees of end vertices of each edge. To compute the above results, we define eight partitions of edge set $E(S P[n])$ and compute their cardinalities in the following way:

$$
\begin{aligned}
E_{1}(S P[n]) & =\{\widehat{e} \hat{f} \in E(S P[n]) \mid \Omega(\widehat{e})=1, \Omega(\widehat{f})=2\}, \\
\left|E_{1}(S P[n])\right| & =2 n+1, \\
E_{2}(S P[n]) & =\{\widehat{e} \widehat{f} \in E(S P[n]) \mid \Omega(\widehat{e})=1, \Omega(\widehat{f})=3\}, \\
\left|E_{2}(S P[n])\right| & =9 n+1, \\
E_{3}(S P[n]) & =\{\widehat{e} \widehat{f} \in E(S P[n]) \mid \Omega(\widehat{e})=1, \Omega(\widehat{f})=4\}, \\
\left|E_{3}(S P[n])\right| & =n, \\
E_{4}(S P[n]) & =\{\widehat{e} \widehat{f} \in E(S P[n]) \mid \Omega(\widehat{e})=2, \Omega(\widehat{f})=2\}, \\
\left|E_{4}(S P[n])\right| & =5 n+4, \\
E_{5}(S P[n]) & =\{\widehat{e} \widehat{f} \in E(S P[n]) \mid \Omega(\widehat{e})=2, \Omega(\widehat{f})=3\}, \\
\left|E_{5}(S P[n])\right| & =18 n-1, \\
E_{6}(S P[n]) & =\{\widehat{e} \hat{f} \in E(S P[n]) \mid \Omega(\widehat{e})=2, \Omega(\widehat{f})=4\}, \\
\left|E_{6}(S P[n])\right| & =2 n, \\
E_{7}(S P[n]) & =\{\widehat{e} \widehat{f} \in E(S P[n]) \mid \Omega(\widehat{e})=3, \Omega(\widehat{f})=3\}, \\
\left|E_{7}(S P[n])\right| & =16 n, \\
E_{8}(S P[n]) & =\{\widehat{e} \widehat{f} \in E(S P[n]) \mid \Omega(\widehat{e})=3, \Omega(\widehat{f})=4\}, \\
\left|E_{8}(S P[n])\right| & =n .
\end{aligned}
$$

Now using equations (1)-(7), we have the following results. 


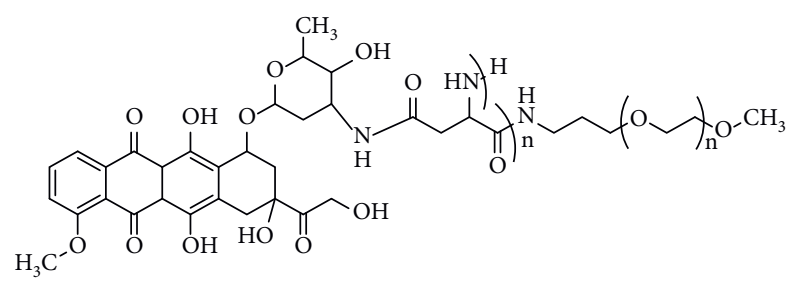

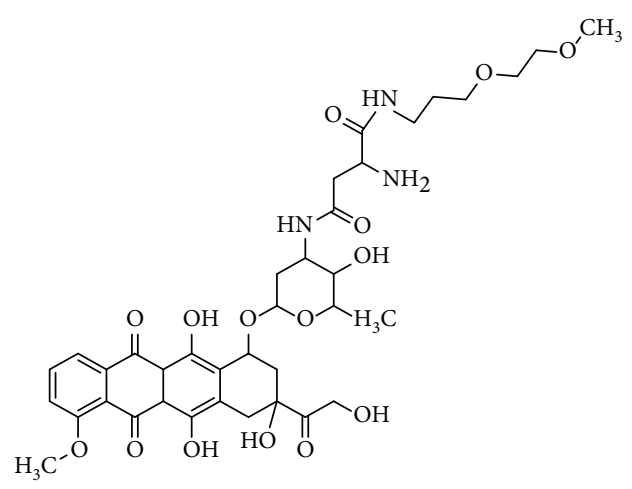

Figure 5: (a) The smart polymer $S P[n]$. (b) The smart polymer $S P[1]$.

\subsubsection{Hyper-Zagreb Index}

$$
\begin{aligned}
H M(G)= & \sum_{\widehat{e} f \in E(G)}[\Omega(\widehat{e})+\Omega(\widehat{f})]^{2}, \\
H M(S P[n])= & \sum_{\widehat{e} \hat{f} \in E_{1}}[\Omega(\widehat{e})+\Omega(\widehat{f})]^{2}+\sum_{\widehat{e} \widehat{f} \in E_{2}}[\Omega(\widehat{e})+\Omega(\widehat{f})]^{2}+\sum_{\widehat{e} \widehat{f} \in E_{3}}[\Omega(\widehat{e})+\Omega(\widehat{f})]^{2}+\sum_{\widehat{e} \widehat{f} \in E_{4}}[\Omega(\widehat{e})+\Omega(\widehat{f})]^{2} \\
& +\sum_{\widehat{e} f \hat{f} \in E_{5}}[\Omega(\widehat{e})+\Omega(\widehat{f})]^{2}+\sum_{\widehat{e} \widehat{e} \in E_{6}}[\Omega(\widehat{e})+\Omega(\widehat{f})]^{2}+\sum_{\widehat{e} \widehat{f} \in E_{7}}[\Omega(\widehat{e})+\Omega(\widehat{f})]^{2}+\sum_{\widehat{e} \widehat{f} \in E_{8}}[\Omega(\widehat{e})+\Omega(\widehat{f})]^{2} \\
= & 9\left|E_{1}\right|+16\left|E_{2}\right|+25\left|E_{3}\right|+16\left|E_{4}\right|+25\left|E_{5}\right|+36\left|E_{6}\right|+36\left|E_{7}\right|+49\left|E_{8}\right| \\
= & 9(2 n+1)+16(9 n+1)+25 n+16(5 n+4)+25(18 n-1)+72 n+36(16 n)+49 n .
\end{aligned}
$$

\subsubsection{First and Second Multiple Zagreb Index}

$$
\begin{aligned}
& P M_{1}(G)=\prod_{\widehat{e} f \in E(G)}[\Omega(\widehat{e})+\Omega(\widehat{f})], \\
& P M_{1}(S P[n])=\prod_{\widehat{e} \widehat{f} \in E_{1}}[\Omega(\widehat{e})+\Omega(\widehat{f})] \times \prod_{\widehat{e} \widehat{f} \in E_{2}}[\Omega(\widehat{e})+\Omega(\widehat{f})] \times \prod_{\widehat{e} \widehat{f} \in E_{3}}[\Omega(\widehat{e})+\Omega(\widehat{f})] \times \prod_{\widehat{e} f \in E_{4}}[\Omega(\widehat{e})+\Omega(\widehat{f})] \\
& \times \prod_{\widehat{e} \hat{f} \in E_{5}}[\Omega(\widehat{e})+\Omega(\widehat{f})] \times \prod_{\widehat{e} \widehat{f} \in E_{6}}[\Omega(\widehat{e})+\Omega(\widehat{f})] \times \prod_{\widehat{e} \hat{e} \in E_{7}}[\Omega(\widehat{e})+\Omega(\widehat{f})] \times \prod_{\widehat{e} \widehat{f} \in E_{8}}[\Omega(\widehat{e})+\Omega(\widehat{f})] \\
& =3^{\left|E_{1}\right|} \times 4^{\left|E_{2}\right|} \times 5^{\left|E_{3}\right|} \times 4^{\left|E_{4}\right|} \times 5^{\left|E_{5}\right|} \times 6^{\left|E_{6}\right|} \times 6^{\left|E_{7}\right|} \times 7^{\left|E_{8}\right|} \\
& =3^{(2 n+1)} \times 4^{(9 n+1)} \times 5^{n} \times 4^{(5 n+4)} \times 5^{(18 n-1)} \times 6^{2 n} \times 6^{16 n} \times 7^{n}, \\
& P M_{2}(G)=\prod_{\widehat{e} \hat{f} \in E(G)}[\Omega(\widehat{e}) \times \Omega(\widehat{f})], \\
& P M_{2}(S P[n])=\prod_{\widehat{e} \hat{f} \in E_{1}}[\Omega(\widehat{e}) \times \Omega(\widehat{f})] \times \prod_{\widehat{e} \widehat{f} \in E_{2}}[\Omega(\widehat{e}) \times \Omega(\widehat{f})] \times \prod_{\widehat{e} \widehat{f} \in E_{3}}[\Omega(\widehat{e}) \times \Omega(\widehat{f})] \times \prod_{\widehat{e} \widehat{f} \in E_{4}}[\Omega(\widehat{e}) \times \Omega(\widehat{f})] \\
& \times \prod_{\widehat{e} \widehat{f} \in E_{5}}[\Omega(\widehat{e}) \times \Omega(\widehat{f})] \times \prod_{\widehat{e} \widehat{f} \in E_{6}}[\Omega(\widehat{e}) \times \Omega(\widehat{f})] \times \prod_{\widehat{e} \widehat{f} \in E_{7}}[\Omega(\widehat{e}) \times \Omega(\widehat{f})] \times \prod_{\widehat{e} \widehat{f} \in E_{8}}[\Omega(\widehat{e}) \times \Omega(\widehat{f})] \\
& =2^{\left|E_{1}\right|} \times 3^{\left|E_{2}\right|} \times 4^{\left|E_{3}\right|} \times 4^{\left|E_{4}\right|} \times 6^{\left|E_{5}\right|} \times 8^{\left|E_{6}\right|} \times 9^{\left|E_{7}\right|} \times 12^{\left|E_{8}\right|} \\
& =2^{(2 n+1)} \times 3^{(9 n+1)} \times 4^{n} \times 4^{(5 n+4)} \times 6^{(18 n-1)} \times 4^{2 n} \times 9^{16 n} \times 12^{n} \text {. }
\end{aligned}
$$




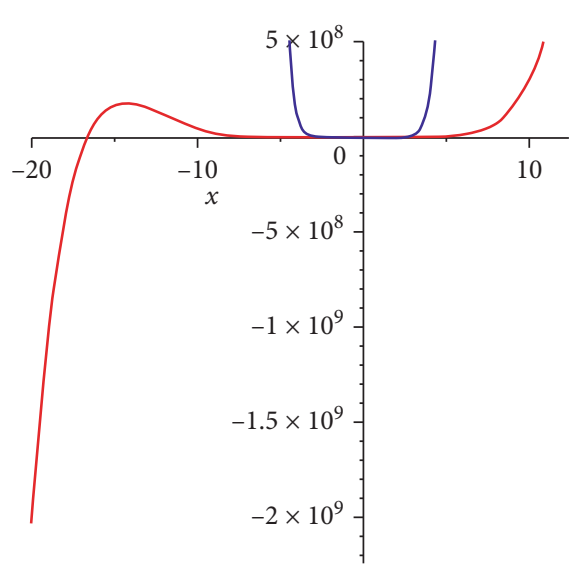

(a)

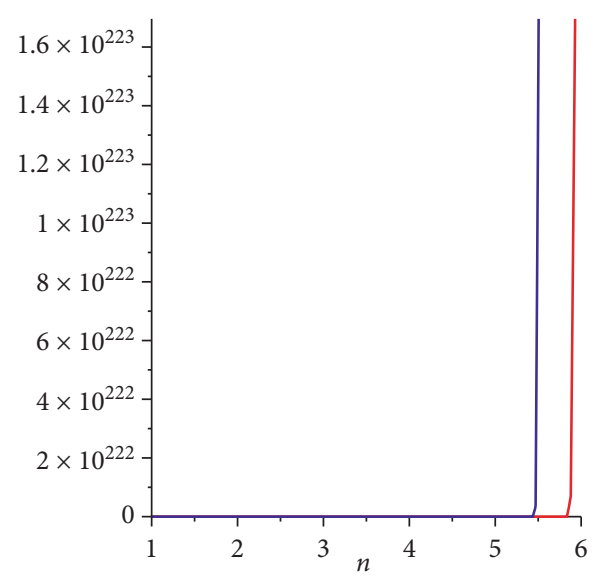

(b)

FIgURE 6: Comparison of topological indices and polynomials for $S P[n]$.

\subsubsection{First and Second Zagreb Polynomial}

$$
\begin{aligned}
M_{1}(G, x)= & \sum_{\widehat{e} f \in E(G)} x^{[\Omega(\widehat{e})+\Omega(\widehat{f})]}, \\
M_{1}(S P[n], x)= & \sum_{\widehat{e} \hat{f} \in E_{1}} x^{[\Omega(\widehat{e})+\Omega(\widehat{f})]}+\sum_{\widehat{e} \widehat{f} \in E_{2}} x^{[\Omega(\widehat{e})+\Omega(\widehat{f})]}+\sum_{\widehat{e} \hat{f} \in E_{3}} x^{[\Omega(\widehat{e})+\Omega(\widehat{f})]}+\sum_{\widehat{e} \hat{f} \in E_{4}} x^{[\Omega(\widehat{e})+\Omega(\widehat{f})]} \\
& +\sum_{\widehat{e} \widehat{f} \in E_{5}} x^{[\Omega(\widehat{e})+\Omega(\widehat{f})]}+\sum_{\widehat{e} \widehat{f} \in E_{6}} x^{[\Omega(\widehat{e})+\Omega(\widehat{f})]}+\sum_{\widehat{e} \widehat{f} \in E_{7}} x^{[\Omega(\widehat{e})+\Omega(\widehat{f})]}+\sum_{\widehat{e} \hat{f} \in E_{8}} x^{[\Omega(\widehat{e})+\Omega(\widehat{f})]} \\
= & (2 n+1) x^{3}+(9 n+1) x^{4}+n x^{5}+(5 n+4) x^{5}+(18 n-1) x^{5}+2 n x^{6}+16 n x^{6}+n x^{7}, \\
M_{2}(G, x)= & \sum_{\widehat{e} f \in E(G)} x^{[\Omega(\widehat{e}) \times \Omega(\widehat{f})]}, \\
M_{2}(S P[n], x)= & \sum_{\widehat{e} \widehat{f} \in E_{1}} x^{[\Omega(\widehat{e}) \times \Omega(\widehat{f})]}+\sum_{\widehat{e} \widehat{f} \in E_{2}} x^{[\Omega(\widehat{e}) \times \Omega(\widehat{f})]}+\sum_{\widehat{e} \hat{f} \in E_{3}} x^{[\Omega(\widehat{e}) \times \Omega(\widehat{f})]}+\sum_{\widehat{e} \hat{f} \in E_{4}} x^{[\Omega(\widehat{e}) \times \Omega(\widehat{f})]} \\
& +\sum_{\widehat{e} \widehat{f} \in E_{5}} x^{[\Omega(\widehat{e}) \times \Omega(\widehat{f})]}+\sum_{\widehat{e} \widehat{f} \in E_{6}} x^{[\Omega(\widehat{e}) \times \Omega(\widehat{f})]}+\sum_{\widehat{e} \widehat{f} \in E_{7}} x^{[\Omega(\widehat{e}) \times \Omega(\widehat{f})]}+\sum_{\widehat{e} \widehat{f} \in E_{8}} x^{[\Omega(\widehat{e}) \times \Omega(\widehat{f})]} \\
= & \left|E_{1}\right| x^{2}+\left|E_{2}\right| x^{3}+\left|E_{3}\right| x^{4}+\left|E_{4}\right| x^{4}+\left|E_{5}\right| x^{6}+\left|E_{6}\right| x^{8}+\left|E_{7}\right| x^{9}+\left|E_{8}\right| x^{12} \\
= & (2 n+1) x^{2}+(9 n+1) x^{3}+n x^{4}+(5 n+4) x^{4}+(18 n-1) x^{6}+2 n x^{8}+16 n x^{9}+n x^{12} .
\end{aligned}
$$

3.2. Graphical Representation and Discussion of Results. In Figure 6(a), comparison of $M_{1}(S P[n], x)$ and $M_{2}(S P[n], x)$ polynomials of $S P[n]$ is given with $M_{1}(S P[n], x)$ in red and $M_{2}(S P[n], x)$ in blue. In Figure 6(b), we compare the indices $P M_{1}(S P[n])$ and $P M_{2}(S P[n])$ with $P M_{1}(S P[n])$ in red and $P M_{2}(S P[n])$ in blue.

\section{Comparison of Topological Indices and Polynomials}

The graphical representations of topological indices $M_{1}(G)$, $M_{2}(G)$, and $H M(G)$ for $G$ equivalent $N_{S C} C_{5}[n], N S D[n]$, and $S P[n]$ are depicted in Figures 2(c), 4(c), and 6(b) for certain values of $n$. For a better view to the reader, we have 
joined all the terms of the sequence points in the graphs by curves, so one can see that the graphs are increasing. By varying the values of $n$, the topological indices behave differently.

The graphical representations of topological indices $P M_{1}(G)$ and $P M_{2}(G)$ for $G$ equivalent $N_{5 C} C_{6}[n]$ and NS D $[n]$ are depicted in Figures 2(b) and 4(b) for certain values of $n$. For a better view to the reader, we have joined all the terms of the sequence points in the graphs by curves, so one can see that the graphs are increasing. One can see that, by varying the values of $n$, the topological indices behave differently.

The comparisons of Zagreb polynomials $M_{1}(G, x)$ and $M_{2}(G, x)$ for $G$ equivalent $N S C_{5} C_{6}[n], N S D[n]$, and $S P[n]$ are depicted in Figures 2(a), 4(a), and 6(a) for certain values of $x$ and for fix $n=10$. We can see from the graphs in Figure 2 that $M_{1}(G, x)$ and $M_{2}(G, x)$ are decreasing and increasing on $(-\infty, 0]$ and $[0, \infty)$, respectively. By varying the values of $x$, the polynomials behave differently. In Figure 4, the $M_{1}(G, x)$ and $M_{2}(G, x)$ behave differently and $M_{1}(N S D[n], x)$ is decreasing and increasing on $(-\infty, 0]$ and $[0, \infty)$, respectively, whereas $M_{2}(N S D[n], x)$ is increasing on $(-\infty, \infty)$. In Figure 6 , the $M_{1}(G, x)$ and $M_{2}(G, x)$ behave differently and $M_{1}(S P[n], x)$ is decreasing and increasing on $[-14,0]$ and $(-\infty, 14] \cup[0, \infty)$, respectively, whereas $M_{2}(S P[n], x)$ is decreasing and increasing on $(-\infty, 0]$ and $[0, \infty)$.

\section{Conclusion}

In this paper, we have managed nanostar dendrimers and star polymer and concentrated their topological records. We decide first and second Zagreb files, hyper-Zagreb record, first different Zagreb file, second numerous Zagreb file, and Zagreb polynomials for nanostar dendrimer and smart polymer. Toward the end, we give a graphical portrayal of all records and polynomials. In future, we are intrigued to register topological records for some new concoction diagrams.

\section{Data Availability}

The data used to support the findings of this study are available from the corresponding author upon request.

\section{Conflicts of Interest}

The authors declare that there are no conflicts of interest regarding the publication of this paper.

\section{Acknowledgments}

This research was supported by the Higher Education Commission of Pakistan under NRPU project "Properties of Ranking Ideals" via Grant no. 20-3665/R\&D/HEC/14/699.

\section{References}

[1] M. V. Diudea and G. Katona, "Molecular topology of dendrimers," in Advances in Dendritic Macromolecules, G. A. Newkome, Ed., vol. 4, pp. 135-201, 1999.
[2] H. Wiener, "Structural determination of paraffin boiling points," Journal of the American Chemical Society, vol. 69, no. 1, pp. 17-20, 1947.

[3] A. A. Dobrynin, R. Entringer, and I. Gutman, "Wiener index of trees: theory and applications," Acta Applicandae Mathematicae, vol. 66, no. 3, pp. 211-249, 2001.

[4] I. Gutman and O. E. Polansky, Mathematical Concepts in Organic Chemistry, Springer-Verlag, Berlin, Germany, 1986.

[5] I. Gutman and N. Trinajstić, "Graph theory and molecular orbitals. Total $\varphi$-electron energy of alternant hydrocarbons," Chemical Physics Letters, vol. 17, no. 4, pp. 535-538, 1972.

[6] G. H. Shirdel, H. RezaPour, and A. M. Sayadi, "The hyperzagreb index of graph operations," Iranian Journal of Mathematical Chemistry, vol. 4, no. 2, pp. 213-220, 2013.

[7] M. Ghorbani and N. Azimi, "Note on multiple Zagreb indices," Iranian Journal of Mathematical Chemistry, vol. 3, no. 2, pp. 137-143, 2012.

[8] I. Gutman and K. C. Das, "Some properties of the second Zagreb index," MATCH Communications in Mathematical and in Computer, vol. 50, pp. 103-112, 2004.

[9] M. Bača, J. Horváthová, M. Mokrišová, and A. Suhányiová, "On topological indices of fullerenes," Applied Mathematics and Computation, vol. 251, pp. 154-161, 2015.

[10] M. Bača, J. Horváthová, M. Mokrišová, A. SemanicovFenovckov, and A. Suhányiová, "On topological indices of a carbon nanotube network," Canadian Journal of Chemistry, vol. 93, no. 10, pp. 1157-1160, 2015.

[11] J.-B. Liu, C. Wang, S. Wang, and B. Wei, "Zagreb indices and multiplicative Zagreb indices of eulerian graphs," Bulletin of the Malaysian Mathematical Sciences Society, vol. 42, no. 1, pp. 67-78, 2019.

[12] J.-B. Liu, X.-F. Pan, F.-T. Hu, and F.-F. Hu, "Asymptotic Laplacian-energy-like invariant of lattices," Applied Mathematics and Computation, vol. 253, pp. 205-214, 2015.

[13] J.-B. Liu and X.-F. Pan, "Minimizing Kirchhoff index among graphs with a given vertex bipartiteness," Applied Mathematics and Computation, vol. 291, pp. 84-88, 2016.

[14] J. B. Liu, J. Zhao, and Z. Zhu, "On the number of spanning trees and normalized Laplacian of linear octagonal-quadrilateral networks," International Journal of Quantum Chemistry, vol. 119, no. 17, Article ID e25971, 2019.

[15] W. Gao, M. K. Siddiqui, M. Imran, M. K. Jamil, and M. R. Farahani, "Forgotten topological index of chemical structure in drugs," Saudi Pharmaceutical Journal, vol. 24, no. 3, pp. 258-264, 2016.

[16] W. Gao and M. K. Siddiqui, "Molecular descriptors of nanotube, oxide, silicate, and triangulene networks," Journal of Chemistry, vol. 2017, Article ID 6540754, 10 pages, 2017.

[17] W. Gharibi, A. Ahmad, and M. K. Siddiqui, "On Zagreb indices, Zagreb polynomials of nanocone and nanotubes," Journal of Computational and Theoretical Nanoscience, vol. 13, no. 8, pp. 5086-5092, 2016.

[18] I. Gutman, "Degree-based topological indices," Croatica Chemica Acta, vol. 86, no. 4, pp. 351-361, 2013.

[19] M. Imran, S. Hayat, and M. Y. H. Mailk, "On topological indices of certain interconnection networks," Applied Mathematics and Computation, vol. 244, pp. 936-951, 2014.

[20] M. Imran, M. Siddiqui, M. Naeem, and M. Iqbal, "On topological properties of symmetric chemical structures," Symmetry, vol. 10, no. 5, p. 173, 2018.

[21] M. K. Siddiqui, M. Imran, and A. Ahmad, "On Zagreb indices, Zagreb polynomials of some nanostar dendrimers," Applied Mathematics and Computation, vol. 280, pp. 132-139, 2016. 
[22] M. K. Siddiqui and W. Gharibi, "On Zagreb indices, Zagreb polynomials of mesh derived networks," Journal of Computational and Theoretical Nanoscience, vol. 13, no. 11, pp. 8683-8688, 2016.

[23] M. K. Siddiqui, M. Naeem, N. A. Rahman, and M. Imran, "Computing topological indicesof certain networks," Journal Optoelectronics and Advanced Materials, vol. 18, no. 9, pp. 884-892, 2016.

[24] M. Rostamia, M. Habanianb, and H. Moghanianc, "Some topological indices for theoretical study of two types of Nanostar Denderimers," Digest Journal of Nanomaterials and Biostructures, vol. 7, no. 1, pp. 247-252, 2012.

[25] J. Singh and K. A. Tahami, "Smart polymer based delivery systems for peptides and proteins," Recent Patents on Drug Delivery and Formulation, vol. 1, no. 1, pp. 65-71, 2007.

[26] R. Freitag, Synthetic Polymers for Biotechnology and Medicine, Biotechnology Intelligence Unit 4, CRC Press, Boca Raton, FL, USA, 2003.

[27] S. S. Kulkarni and N. H. Aloorkar, "Smart polymers in drug delivery: an overview," Journal of Pharmacy Research, vol. 3, no. 1, pp. 100-108, 2010.

[28] K. Osada, R. J. Christie, and K. Kataoka, "Polymeric micelles from poly(ethylene glycol)-poly(amino acid) block copolymer for drug and gene delivery," Journal of The Royal Society Interface, vol. 6, pp. 325-339, 2009.

[29] B. S. Shettya, V. Lokeshab, P. S. Ranjinic, and K. C. Dasd, "Computing some topological indices of smart polymer," Digest Journal of Nanomaterials and Biostructures, vol. 7, no. 3, pp. 1097-1102, 2012. 